\title{
Bioorthogonal Fluorescent Nanodiamonds for Continuous Long-Term Imaging and Tracking of Membrane Proteins
}

Feng-Jen Hsieh, ${ }^{\mathrm{a}-\mathrm{c}, \ddagger}$ Shingo Sotoma, ${ }^{\mathrm{a}, \uparrow, \uparrow}$ Hsin-Hung Lin, ${ }^{\mathrm{a}}$ Ching-Ya Cheng, ${ }^{\mathrm{a}}$ Tsyr-Yan Yu, ${ }^{\mathrm{a}, \mathrm{b}}$ Chia-Lung Hsieh, ${ }^{a}$ Chun-Hung Lin ${ }^{\mathrm{b}-\mathrm{d}, *}$ and Huan-Cheng Chang ${ }^{\mathrm{a}, \mathrm{e}, \mathrm{f}, *}$

${ }^{a}$ Institute of Atomic and Molecular Sciences, Academia Sinica, Taipei 106, Taiwan

b Taiwan International Graduate Program - Chemical Biology and Molecular Biophysics, Academia Sinica, Taipei, 115, Taiwan

${ }^{c}$ Institute of Biochemical Sciences, National Taiwan University, Taipei, 106, Taiwan

${ }^{\mathrm{d}}$ Institute of Biological Chemistry, Academia Sinica, Taipei, 115, Taiwan

e Department of Chemical Engineering, National Taiwan University of Science and Technology, Taipei 106, Taiwan

${ }^{\mathrm{f}}$ Department of Chemistry, National Taiwan Normal University, Taipei 106, Taiwan

$\dagger$ Current address: Institute for Protein Research, Osaka University, Suita-shi, Osaka 565-0871, Japan

$\$$ These authors contributed equally to this work.

* Corresponding authors: C.-H.L. (hunhung@gate.sinica.edu.tw) or H.-C.C. (hchang@gate.sinica.edu.tw) 


\section{Experimental sections}

Preparation and surface modification of FNDs. The method of preparing FNDs has been previously described. ${ }^{1}$ Briefly, $\sim 100 \mathrm{~nm}$ diamond powders (Element Six, USA) were irradiated by a $40 \mathrm{keV}$ helium beam, annealed in vacuum at $800{ }^{\circ} \mathrm{C}$ for $2 \mathrm{~h}$, and oxidized in air at $450{ }^{\circ} \mathrm{C}$ for $2 \mathrm{~h}$ to produce FNDs. Microwave-assisted acid treatment $\left(100 \mathrm{~W}, 100{ }^{\circ} \mathrm{C}, 3 \mathrm{~h}\right)$ in concentrated $\mathrm{H}_{2} \mathrm{SO}_{4}$ and $\mathrm{HNO}_{3}(3: 1$ in volume ratio) was followed to remove metallic contaminants and concurrently improve the dispersibility of FNDs in water. After washing 3 times with deionized distilled water (DDW), the FND particles were dried and stored before use. To prepare alkyne-HPGFNDs, FNDs $(5 \mathrm{mg})$ were added to glycidol ( $2 \mathrm{~mL}$; SigmaAldrich, USA $)$ and heated at $120^{\circ} \mathrm{C}$ for $2 \mathrm{~h}$ under nitrogen atmosphere. Glycidyl propargyl ether $(1 \mathrm{~mL}$; TCI Chemicals, Japan) was then mixed with the suspension and continually reacted for another $22 \mathrm{~h}$ under the same conditions. Finally, the alkyne-HPGFND particles were washed 2 times with methanol and 3 times with DDW to remove unbound polymers. A similar protocol was used to prepare HPGFNDs, i.e. the $\mathrm{FND} /$ glycidol mixture was heated at $120{ }^{\circ} \mathrm{C}$ for $24 \mathrm{~h}$ under nitrogen without the addition of glycidyl propargyl ether. The concentrations of both particles in the solution was determined by measuring the fluorescence intensity with reference to the calibration curve of bare FNDs.

Characterization of bare and surface-modified FNDs. Hydrodynamic sizes of bare and surface-modified FNDs were measured with the DelsaNano C particle size and zeta potential analyzer (Beckman Coulter, USA). Samples for infrared spectroscopic measurements were prepared in KBr-pellets (Sigma-Aldrich, USA) and the spectra were obtained with the Bruker VERTEX 70 FTIR spectrometer (Bruker, Germany) in the wavenumber range between 4000 and $500 \mathrm{~cm}^{-1}$ (resolution: $4 \mathrm{~cm}^{-1}$ ). Thermogravimetric analysis (TGA) was conducted with the STA PT1000 TG-DSC simultaneous thermal analyzer (Linseis, USA) for samples in air from room temperature to $800{ }^{\circ} \mathrm{C}$ with a heating rate of $5{ }^{\circ} \mathrm{C} / \mathrm{min}$. For two-dimensional NMR spectroscopy, surface-modified FNDs were dispersed in $\mathrm{D}_{2} \mathrm{O}$ with a concentration of $1 \mathrm{mg} / \mathrm{mL}$. Experiments were carried out at $25^{\circ} \mathrm{C}$ on the Avance III HD NMR spectrometer (Bruker, USA) operating at $850 \mathrm{MHz}$ (proton frequencies). The spectrometer was equipped with a triple resonance (TCI) probe and a standard NMR pulse sequence, hsqcetgpsisp.2, was used to record heteronuclear single quantum coherence (HSQC) spectra. The cross peaks in a ${ }^{1} \mathrm{H}_{-}{ }^{13} \mathrm{C}$ HSQC spectrum are generally associated with one-bond $\mathrm{H}-$ $\mathrm{C}$ pair. In the case of alkynes, the one-bond and two-bond $\mathrm{C}-\mathrm{H}$ couplings are around $240 \mathrm{~Hz}$ and $50 \mathrm{~Hz}$, respectively. A large two-bond coupling has been documented to be sufficient to produce a cross peak in the HSQC spectrum. ${ }^{2}$

Click chemistry and quantification of alkynyl groups. Click chemistry between alkyne-HPGFNDs and Alexa Fluor 488-azides was conducted following the protocols of Presolski et al. ${ }^{3}$ with slight modifications. Briefly, alkyne-HPGFNDs $(100 \mu \mathrm{g})$ were added into Alexa Fluor 488-azide solution (100 $\mu \mathrm{L}$; Thermo Fisher Scientific, USA) containing $0.15 \mathrm{mM}$ Tris(3-hydroxypropyltriazolylmethyl)amine (THPTA; TCI Chemicals, Japan), $0.03 \mathrm{mM} \mathrm{CuSO}_{4}$ (Sigma-Aldrich, USA), $5 \mathrm{mM}$ aminoguanidine (TCI Chemicals, Japan), and $5 \mathrm{mM}$ sodium ascorbate (Sigma-Aldrich, USA) as the catalysts. After 1-h 
incubation, the Alexa Fluor 488-azide-bound alkyne-HPGFND particles were washed 5 times with DDW, collected by centrifugation at 15,000 rpm for $5 \mathrm{~min}$, and re-dispersed in DDW $(100 \mu \mathrm{L})$. The fluorescence intensities (excitation at $475 \mathrm{~nm} / 520 \mathrm{~nm}$ and emission at 500-550 nm/660-720 nm for Alexa Fluor 488/FND, respectively) of each sample were measured with the GloMax discover multimode microplate reader (Promega, USA) to obtain the Alexa Fluor 488 and FND concentrations. The number of alkynyl groups per alkyne-HPGFND was then estimated under the assumption that FNDs are spherical in shape.

Extraction of azide-modified proteins and MALDI-TOF MS analysis. Azide-modified biotin-conjugated NeutraAvidin (NA/AB) was prepared by mixing N-[2-[2-[2-(2-azidoethoxy)ethoxy]ethoxy]ethyl] biotinamide (100 mg; Biotin-PEG3-Azide, TCI Chemicals, Japan) and NeutrAvidin (10 mg; Thermo Fisher Scientific, USA) in DDW together for $30 \mathrm{~min}$. The conjugates were then purified by membrane filtration (Amicon Ultra-0.5 mL $10 \mathrm{~K}$ Centrifugal Filters, Millipore, USA), after which the NA/AB concentration was measured with the NanoDrop 2000 spectrophotometer (Thermo Fisher Scientific, USA) and adjusted with DDW to be $0.01 \mathrm{mM}$. A stock solution composed of $1 \mathrm{mM}$ bovine serum albumin (BSA; Sigma-Aldrich, USA) in phosphate-buffered saline (PBS; Gibco, USA) was prepared for further use.

The extraction of NA/AB by alkyne-HPGFNDs followed the click chemistry protocols described in the previous section. Briefly, alkyne-HPGFNDs $(100 \mu \mathrm{g})$ were added to a BSA:NA/AB mixture $(100: 1$ in molar ratio) in PBS $(100 \mu \mathrm{L})$ with the catalysts (i.e., $1.5 \mathrm{mM}$ THPTA, $0.3 \mathrm{mM} \mathrm{CuSO}_{4}, 5 \mathrm{mM}$ aminoguanidine, and $5 \mathrm{mM}$ sodium ascorbate). After 1-h incubation, the alkyne-HPGFND particles were washed 5 times with PBS $(200 \mu \mathrm{L})$, collected by centrifugation at $15,000 \mathrm{rpm}$ for $5 \mathrm{~min}$, and analyzed by matrix-assisted laser desorption/ionization time-of-flight mass spectrometry (MALDI-TOF MS). In this analysis, each sample (e.g., FND pellets after the NA/AB extraction) was first mixed with an acetonitrile/ $\mathrm{H}_{2} \mathrm{O}$ solution $(2 / 1$ in volume ratio, $1 \mu \mathrm{L}$ ) containing $20 \mathrm{mg} / \mathrm{mL}$ 2,5-dihydroxybenzoic acid (Sigma-Aldrich, USA) and $0.1 \%$ trifluoroacetic acid (TFA; Sigma-Aldrich, USA), and then transferred to an aluminum plate and analyzed with the Microflex LRF mass spectrometer (Bruker, Germany). The laser power used for the measurement was adjusted slightly larger than the desorption/ionization threshold of the analyte. Each MS spectrum was collected from $\sim 200$ laser shots randomly scanned across the plate surface.

Cell culture and cell viability assays. HeLa cells or HFW cells were cultured in Dulbecco's modified Eagle's medium (Gibco, USA) supplemented with 10\% fetal calf serum (Gibco, USA) and 1\% antibioticantimycotic (Gibco, USA) at $37{ }^{\circ} \mathrm{C}$ with $5 \% \mathrm{CO}_{2}$. Alexa Fluor 488-conjugated wheat germ agglutinin (Thermo Fisher Scientific, USA) was used for cell membrane staining when needed. To inspect the cytotoxicity of alkyne-HPGFNDs, cells were seeded in 96-well plates $\left(3 \times 10^{3}\right.$ cells/well $) 1$ day before examination. The culture medium was then replaced by medium containing $50 \mathrm{~nm}$ or $100 \mathrm{~nm}$ alkyneHPGFNDs with the concentration varying from $0.1,0.5,1$, to $2 \mathrm{mg} / \mathrm{mL}$. After 7-h incubation at $37^{\circ} \mathrm{C}$ with $5 \% \mathrm{CO}_{2}$, dehydrogenase activities of the cells were measured by the cell counting kit-8 (CCK-8, Dojindo, Japan) following the instructions of the manufacturer. 
Labelling of azido-sialoglycoproteins and integrin $\alpha 5$. To perform sialoglycoprotein labelling, cells $(1 \times$ $10^{5}$ cells/well) were seeded in medium containing ManNAz ( $40 \mu \mathrm{M}$; Thermo Fisher Scientific, USA) 2 days before experiments. After removal of culture medium by decantation without washing, alkyne-HPGFNDs $(0.5 \mathrm{mg})$ with the catalysts $\left(3 \mathrm{mM}\right.$ THPTA, $0.6 \mathrm{mM} \mathrm{CuSO}_{4}, 5 \mathrm{mM}$ aminoguanidine, and $5 \mathrm{mM}$ sodium ascorbate in PBS) were added to label azido-sialoglycoprotein on the surface of living cells. ${ }^{4}$ Following incubation for $\sim 30 \mathrm{~min}$, the FND-labelled cells were washed 5 times with culture medium to remove nonspecifically bound FNDs and analyzed by flow cytometry or confocal microscopy.

For the integrin $\alpha 5$ targeting, azide-modified antibody was first prepared by mixing $1 \mathrm{mg} / \mathrm{mL}$ antiintegrin $\alpha 5$ antibody ( $250 \mu \mathrm{L}$; BioLegend, USA) with $6.4 \mathrm{mM}$ azido-PEG4-NHS (11 $\mu \mathrm{L}$; TCI Chemicals, Japan), incubated for $2 \mathrm{~h}$ at room temperature, followed by adding Tris- $\mathrm{HCl}$ buffer ( $50 \mu \mathrm{L}, \mathrm{pH} 8$; SigmaAldrich, USA) to terminate the reaction and obtain azide-modified antibody. The same labelling procedures as described above were applied (except no ManNAz) by first using $2 \mu \mathrm{g} / \mathrm{mL}$ azido-anti-integrin $\alpha 5$ antibody in culture medium for $1 \mathrm{~h}$ and then by $1 \mathrm{mg} / \mathrm{mL}$ alkyne-HPGFND.

Flow cytometry, confocal microscopy and epifluorescence microscopy. Cells were detached by trypsinization, transferred to round-bottom 96 -well plates, and analyzed $(\sim 5,000$ cells $)$ by the FACSArray Bioanalyser flow cytometer (BD Bioscience, USA) equipped with a $532 \mathrm{~nm}$ laser for excitation and a 590 nm long-pass emission filter. Confocal microscopy images were acquired by using the Leica TCS SP- 8 microscope (Leica, Germany) equipped with a live-cell imaging system (LCI, Korea), an oil-immersion objective (63×, NA 1.4; Leica, Germany), a supercontinuum white-light laser (NKT Photonics, Denmark) for excitation and a photomultiplier (PMT) and a hybrid detector (HyD) for detection. Fluorescent dye molecules and FNDs were excited by 488 and $561 \mathrm{~nm}$ light, respectively, and the corresponding fluorescence emissions were detected at $500-550$ and $670-800 \mathrm{~nm}$.

Videos were produced from continuously z-scanned images of the same cells for $1-12 \mathrm{~h}$ and played at a rate of $30 \mathrm{z}$-scanned images per second. Each z-stacked image consisted of $15-30 \mathrm{z}$-scans with a step size of $\sim 0.4 \mu \mathrm{m}$. For epifluorescence imaging, the same microscope system was used but with a mercury lamp and an excitation band-pass filter (BP $515-560 \mathrm{~nm}$ ) for excitation and the iXon Ultra 888 electron multiplying CCD (Oxford Instrument, UK) and an emission long-pass filter (LP $590 \mathrm{~nm}$ ) for detection. The exposure time to obtain the individual images was $30 \mathrm{~ms}$, the pixel size was $206 \mathrm{~nm}$, and each video was produced from continuously recorded images for $5 \mathrm{~min}$ ( $\sim 0.1 \mathrm{~s}$ interval between images).

Quantification of cell-attached FNDs. Azido-sialoglycoproteins and integrin $\alpha 5$ on HFW cells was labelled with alkyne-HPGFNDs as mentioned above. After labelling, the cells $\left(5 \times 10^{4}\right)$ were scraped in DDW (1 $\mathrm{mL}$ ) and lysed by sonication in an ultrasonic cleaner (Branson Ultrasonics, USA). The fluorescence intensities of each sample were then measured by using a home-built fluorescence spectrometer equipped with a magnetic modulation system. ${ }^{5}$ The amounts of FNDs attached to the cells were finally estimated by comparing the measured fluorescence intensities of the cell lysates to that of standard FND solutions. 
Trajectory analysis. The trajectories of membrane proteins moving on cell surface were analyzed with the Fiji and home-coded MATLAB (MathWorks, USA) program. ${ }^{6}$ Valid trajectories (longer than 20 continuous frames) were used for mean square displacement (MSD) analysis of anomalous diffusion as

$$
\operatorname{MSD}(n \Delta t)=4 D(n \Delta t)^{\alpha},
$$

where $n$ is the number of sampling steps, $\Delta t$ is the time interval between steps, $D$ is the diffusion coefficient, and $\alpha$ is the anomaly parameter. ${ }^{7}$

\section{Results and discussion}

Figure S11 shows results of the MSD analysis for the movement trajectories of integrin $\alpha 5$ recorded in Video S1 with $\Delta t=0.104 \mathrm{~s}$ and $n=1-19$. We determined a diffusion coefficient of $D=0.041 \pm 0.032 \mu \mathrm{m}^{2} / \mathrm{s}$ (average \pm standard deviation of the distribution in Figure S11a) and an anomaly parameter of $\alpha=0.74 \pm$ 0.32 (average \pm standard deviation of the distribution in Figure S11b) within this time increment of $n \Delta t<2$ s. It is instructive to compare our findings with previous measurements using different technologies. Adopting $40 \mathrm{~nm}$ antibody-coated gold nanoparticles as light scattering probes, Sheetz and co-workers determined the diffusion coefficients of integrins $\beta 1$ and $\alpha 4$ on mouse 3T3 fibroblasts to span from 0.001 to $0.1 \mu \mathrm{m}^{2} / \mathrm{s}$, depending on the treatments. ${ }^{8,9}$ Using photothermal microscopy to observe individual $5 \mathrm{~nm}$ gold nanoparticles surface-functionalized with nanobodies to achieve monovalent labeling of $\beta 3$ integrins on mouse embryonic fibroblasts, Leduc et al. measured the instantaneous diffusion coefficient (with a moving window of $\sim 1 \mathrm{~s}$ ) of the particles outside focal adhesion sites to range from 0.01 to $0.1 \mu \mathrm{m}^{2} / \mathrm{s}$ over an observation time of $25 \mathrm{~s}^{10}$ Our $D$ values fall within these ranges, suggesting that the labelling with 100 nm alkyne-HPGFNDs do not significantly affect the movement of integrin $\alpha 5$, which has a molecular weight of $\sim 150 \mathrm{kDa},{ }^{11}$ on the surface of HFW cells.

\section{References}

(1) Chang, Y. R.; Lee, H. Y.; Chen, K.; Chang, C. C.; Tsai, D. S.; Fu, C. C.; Lim, T. S.; Tzeng, Y. K.; Fang, C. Y.; Han, C. C.; Chang, H. C.; Fann, W. Mass Production and Dynamic Imaging of Fluorescent Nanodiamonds. Nat. Nanotechnol. 2008, 3, 284-288.

(2) Claridge, T. D. High-Resolution NMR Techniques in Organic Chemistry, Elsevier, 2016.

(3) Presolski, S. I.; Hong, V. P.; Finn, M. G. Copper-Catalyzed Azide-Alkyne Click Chemistry for Bioconjugation, Wiley, 2009.

(4) Li, S.; Wang, L.; Yu, F.; Zhu, Z.; Shobaki, D.; Chen, H.; Wang, M.; Wang, J.; Qin, G.; Erasquin, U. J.; Ren, L.; Wang, Y.; Cai, C. Copper-Catalyzed Click Reaction on/in Live Cells. Chem. Sci. 2017, 8, 2107 2114.

(5) Su, L. J.; Wu, M. S.; Hui, Y. Y.; Chang, B. M.; Pan, L.; Hsu, P. C.; Chen, Y. T.; Ho, H. N.; Huang, Y. H.; Ling, T. Y.; Hsu, H. H.; Chang, H. C. Fluorescent Nanodiamonds Enable Quantitative Tracking of Human Mesenchymal Stem Cells in Miniature Pigs. Sci. Rep. 2017, 7, 45607-45617.

(6) Lin, Y. H.; Chang, W. L.; Hsieh, C. L. Shot-Noise Limited Localization of Single $20 \mathrm{~nm}$ Gold Particles with Nanometer Spatial Precision within Microseconds. Opt. Express 2014, 22, 9159-9170. 
(7) Saxton, M. J.; Jacobson, K. Single-Particle Tracking: Applications to Membrane Dynamics. Annu. Rev. Biophys. Biomol. Struct. 1997, 26, 373-399.

(8) Felsenfeld, D. P.; Choquet, D.; Sheetz, M. P. Ligand Binding Regulates the Directed Movement of $\beta 1$ Integrins on Fibroblasts. Nature 1996, 383, 438-440.

(9) Yauch, R. L.; Felsenfeld, D. P.; Kraeft, S.-K.; Chen, L. B.; Sheetz, M. P.; Hemler, M. E. Mutational Evidence for Control of Cell Adhesion through Integrin Diffusion/Clustering, Independent of Ligand Binding. J. Exp. Med. 1997, 186, 1347-1355.

(10) Leduc, C.; Si, S.; Gautier, J.; Soto-Ribeiro, M.; Wehrle-Haller, B.; Gautreau, A.; Giannone, G.; Cognet, L.; Lounis, B. A Highly Specific Gold Nanoprobe for Live-Cell Single-Molecule Imaging. Nano Lett. 2013, 13, 1489-1494.

(11) Diggins, N. L.; Kang, H.; Weaver, A.; Webb, D. J. $\alpha 5 \beta 1$ Integrin Trafficking and Rac Activation are Regulated by APPL1 in a Rab5-Dependent Manner to Inhibit Cell Migration. J. Cell Sci. 2018, 131, jes207019.

(12) Hsieh, F. J.; Chen, Y. W.; Huang, Y. K.; Lee, H. M.; Lin, C. H.; Chang, H. C. Correlative Light-Electron Microscopy of Lipid-Encapsulated Fluorescent Nanodiamonds for Nanometric Localization of Cell Surface Antigens. Anal. Chem. 2018, 90, 1566-1571.

(13) Zhao, L.; Takimoto, T.; Ito, M.; Kitagawa, N.; Kimura, T.; Komatsu, N. Chromatographic Separation of Highly Soluble Diamond Nanoparticles Prepared by Polyglycerol Grafting. Angew. Chem. Int. Ed. 2011, 50, 1388-1392. 
(a)

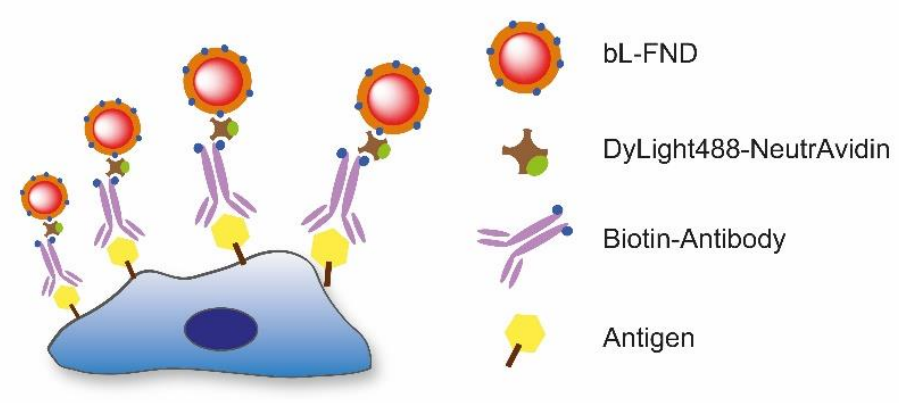

(b)

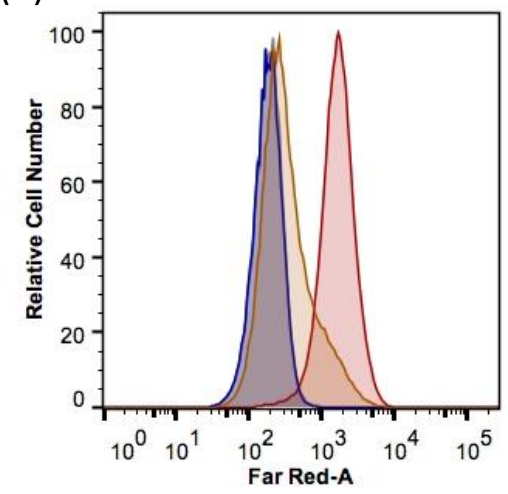

Figure S1. (a) Sandwich immunostaining of antigens on cell surface with biotinylated lipid-encapsulated FNDs (bL-FNDs). ${ }^{12}$ (b) Flow cytometric analysis of HeLa cells labelled with bL-FNDs. The grey region represents the cell-only group, and the red region represents cells labelled with biotin-anti-CD44 antibody, NeutrAvidin, and then bL-FND. The blue and orange regions represent cells labelled by the same method as those in the red region but without NeutrAvidin or biotin-anti-CD44 antibody, respectively. Note that the presence of endogenous biotin on cell surface diminishes the specificity of avidin-biotin-based immunostaining of cells, as evidenced by the orange region in (b).

(a)

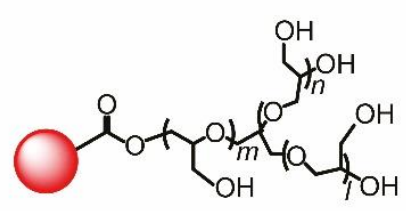

(b)

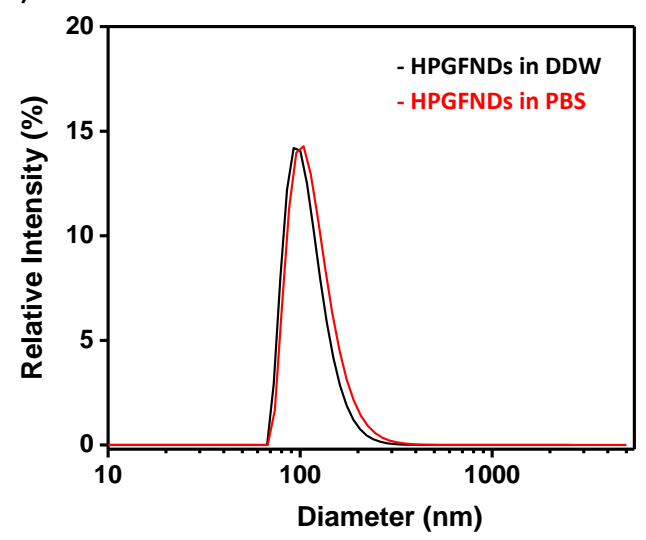

Figure S2. (a) Proposed structure of HPGFND ${ }^{13}$ and (b) hydrodynamic size distributions of HPGFNDs in DDW and PBS. 


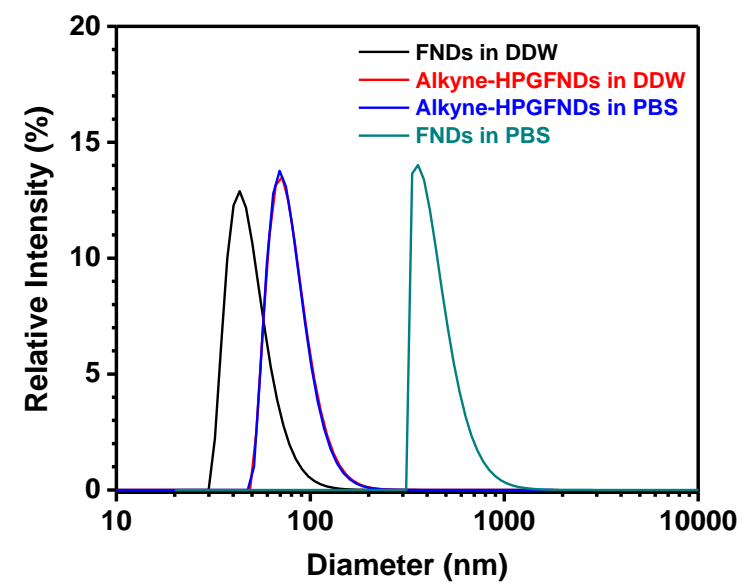

Figure S3. Size distributions of $50 \mathrm{~nm}$ FNDs and alkyne-HPGFNDs in DDW or PBS.

(a)

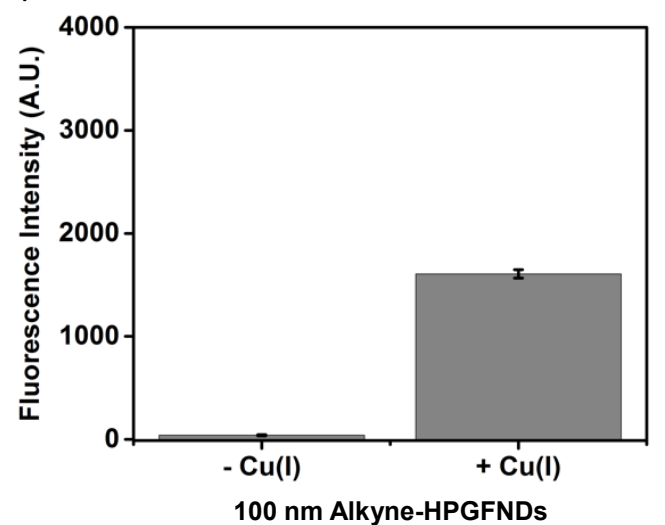

(b)

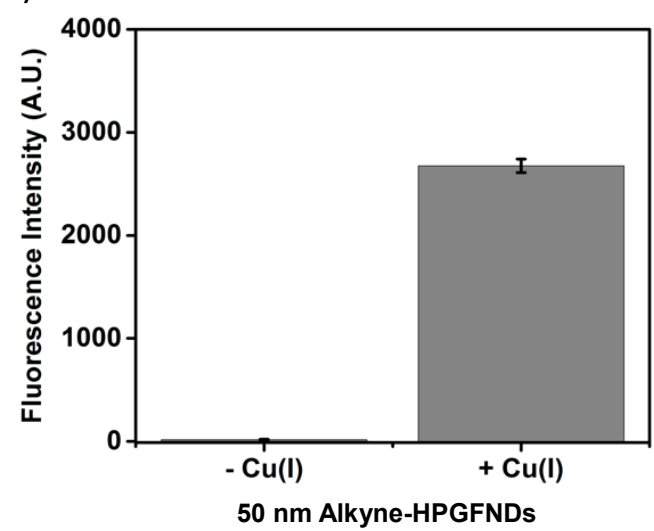

Figure S4. Characterization of the conjugation of alkyne-HPGFNDs with Alexa Fluor 488-azide: (a) 100 $\mathrm{nm}$ and (b) $50 \mathrm{~nm}$ alkyne-HPGFNDs. The fluorescence intensities are normalized with respect to the weight concentration of each sample. 

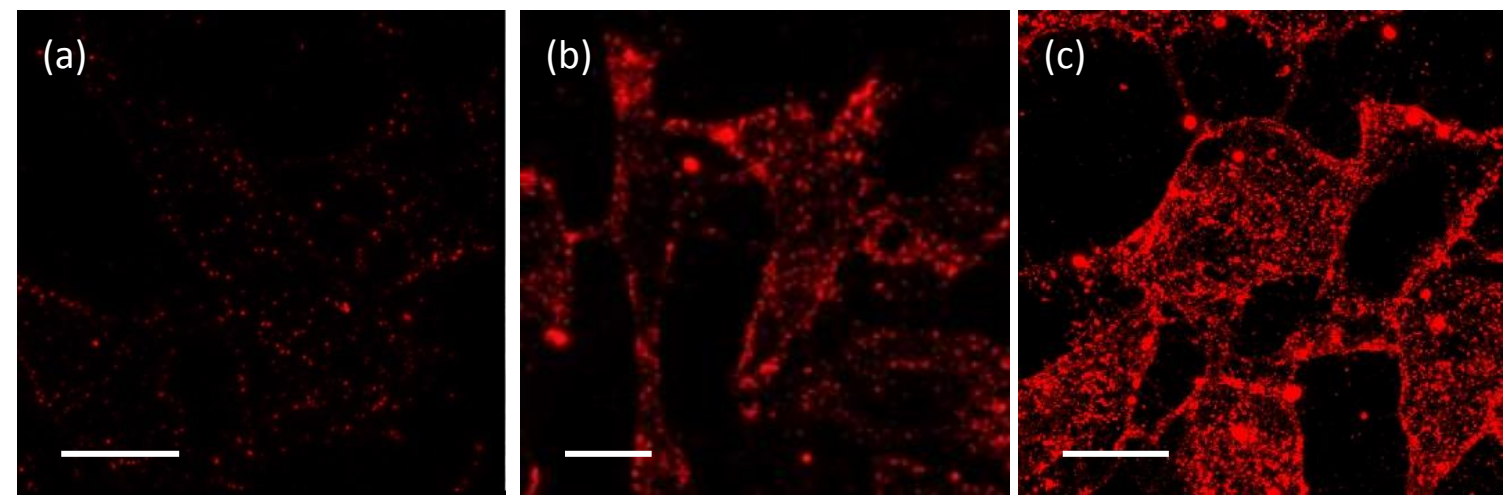

Figure S5. Confocal fluorescence images of azido-sialoglycoprotein-expressing HeLa cells labelled with 100-nm alkyne-HPGFND (1 mg/mL) for (a) $10 \mathrm{~min}$, (b) $20 \mathrm{~min}$, and (c) $30 \mathrm{~min}$. Scale bars: $20 \mu \mathrm{m}$.

(a)

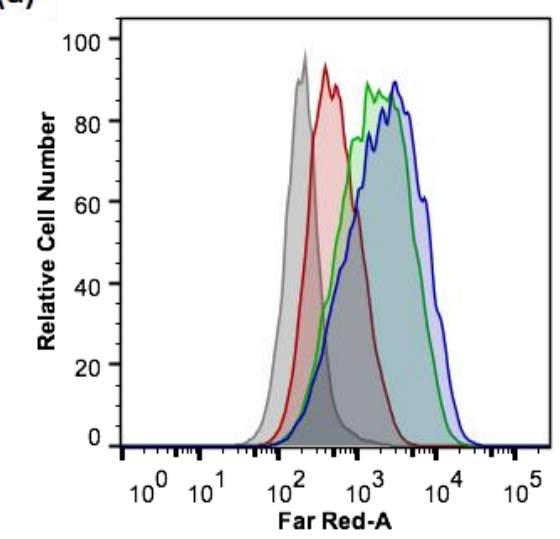

(c)

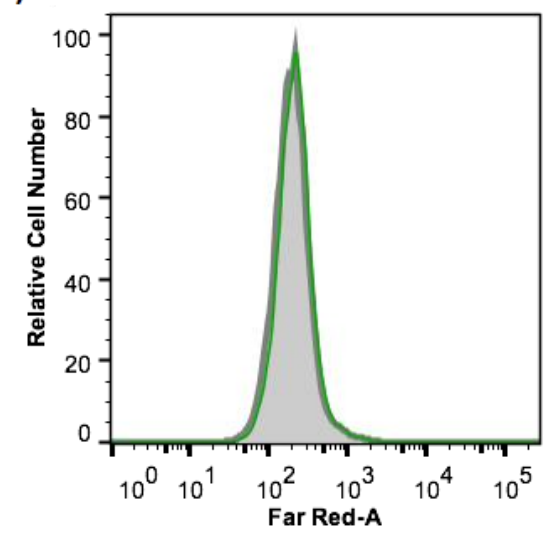

(b)

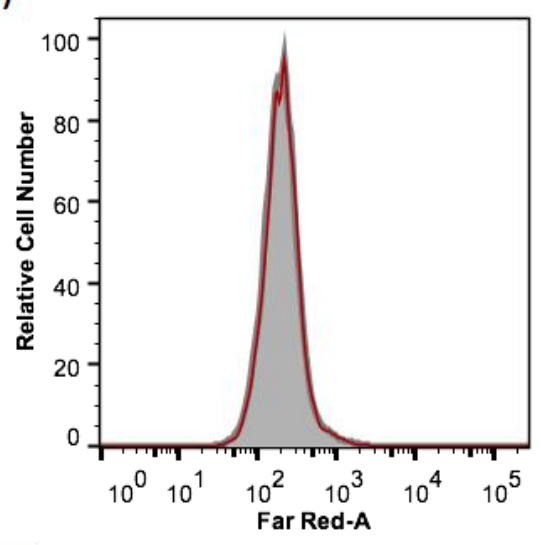

(d)

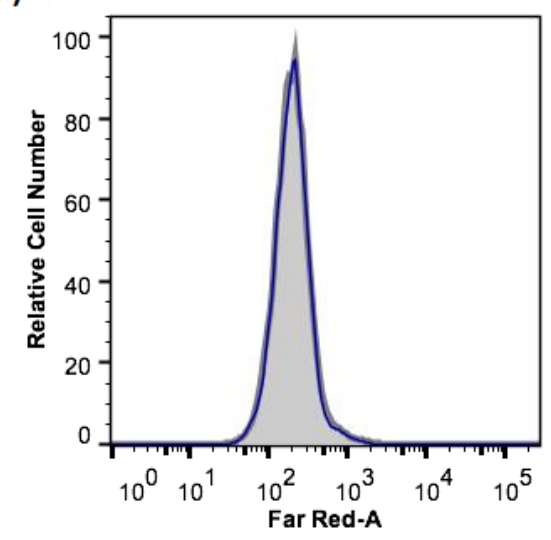

Figure S6. Flow cytometric analysis of azido-sialoglycoproteins on HeLa cells labelled with alkyneHPGFNDs of various concentrations (a) with or (b-d) without $\mathrm{Cu}^{+}$catalyzation. The grey areas represent the cell-only groups, and the red, green and blue curves represent azido-sialoglycoprotein-expressing HeLa cells labelled with $0.1,0.5$, or $1 \mathrm{mg} / \mathrm{mL}$ alkyne-HPGFND, respectively. 

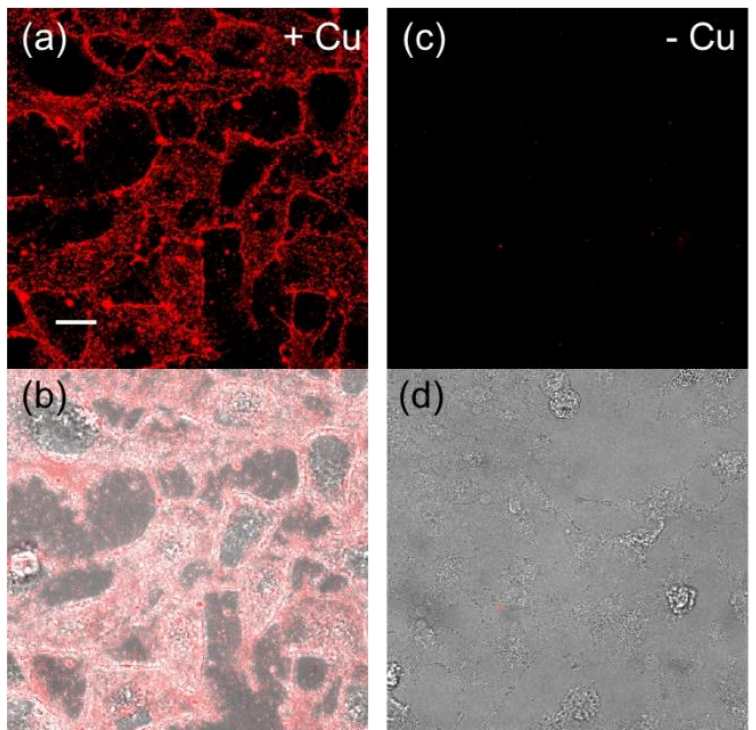

(d)

Figure S7. ( $a, c)$ Fluorescence and (b, d) fluorescence/bright-field merged images of azidosialoglycoprotein-expressing HeLa cells labelled by $100 \mathrm{~nm}$ alkyne-HPGFND $(1 \mathrm{mg} / \mathrm{mL})$ with $(\mathrm{a}, \mathrm{b})$ or without (c, d) $\mathrm{Cu}^{+}$catalyzation. Scale bar: $20 \mu \mathrm{m}$.
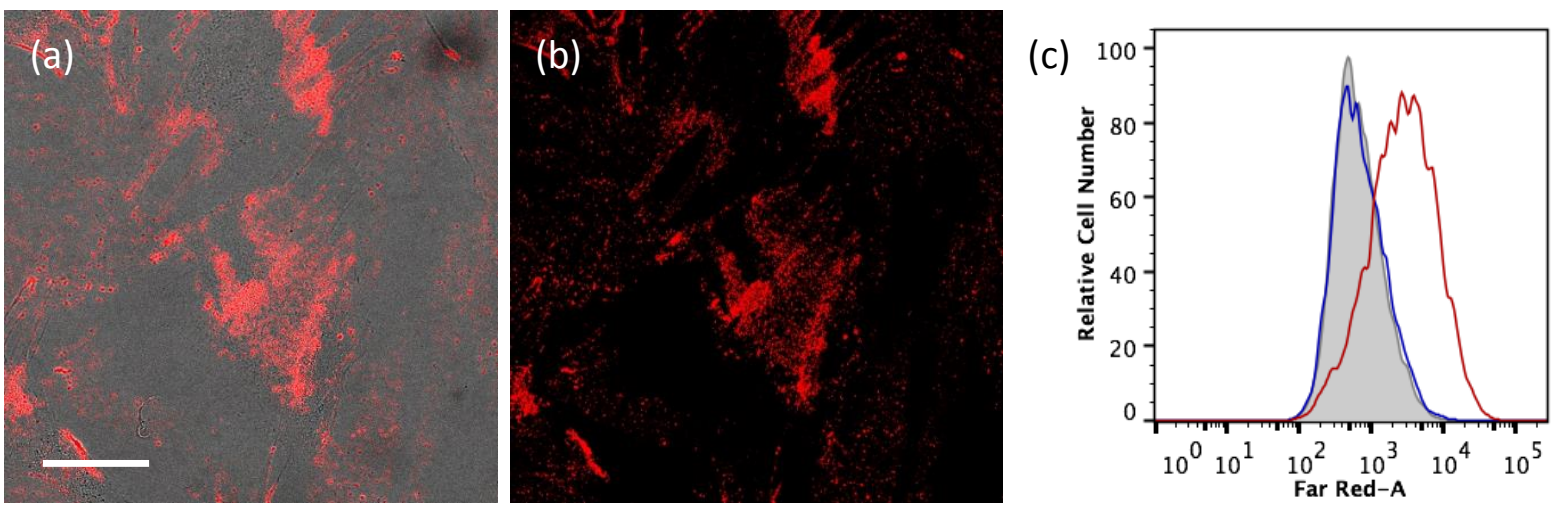

Figure S8. (a) Fluorescence/bright-field merged images and (b) fluorescence images of azidosialoglycoprotein-expressing HFW cells labelled with $1 \mathrm{mg} / \mathrm{mL} 100 \mathrm{~nm}$ alkyne-HPGFND for $30 \mathrm{~min}$. Scale bar: $50 \mu \mathrm{m}$. (c) Flow cytometric analysis of azido-sialoglycoproteins on HFW cells labelled by 100 $\mathrm{nm}$ alkyne-HPGFND $(1 \mathrm{mg} / \mathrm{mL})$ with (red) or without (blue) $\mathrm{Cu}^{+}$catalyzation. The grey area represents the cell-only group. 


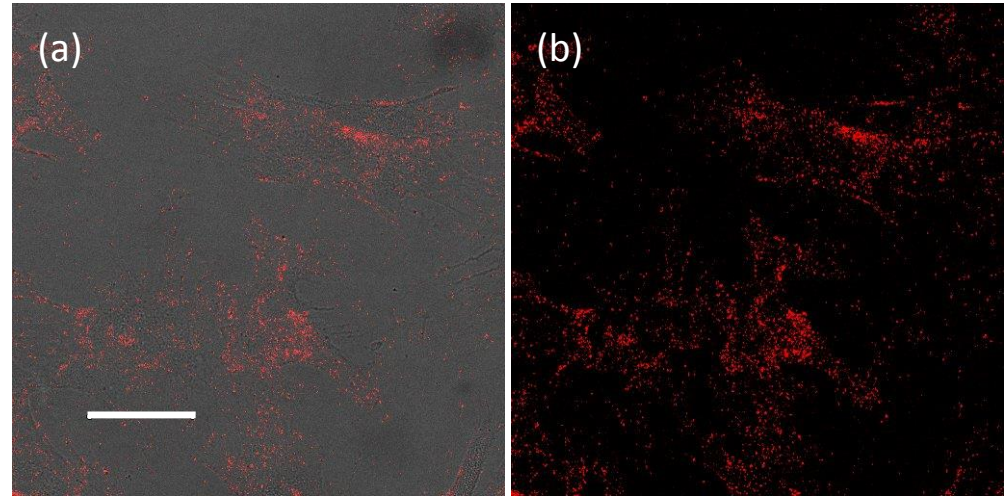

Figure S9. (a) Fluorescence/bright-field merged images and (b) fluorescence images of HFW cells labelled with anti-integrin $\alpha 5$ antibody and then $100 \mathrm{~nm}$ alkyne-HPGFND $(1 \mathrm{mg} / \mathrm{mL})$. Scale bar: $50 \mu \mathrm{m}$.

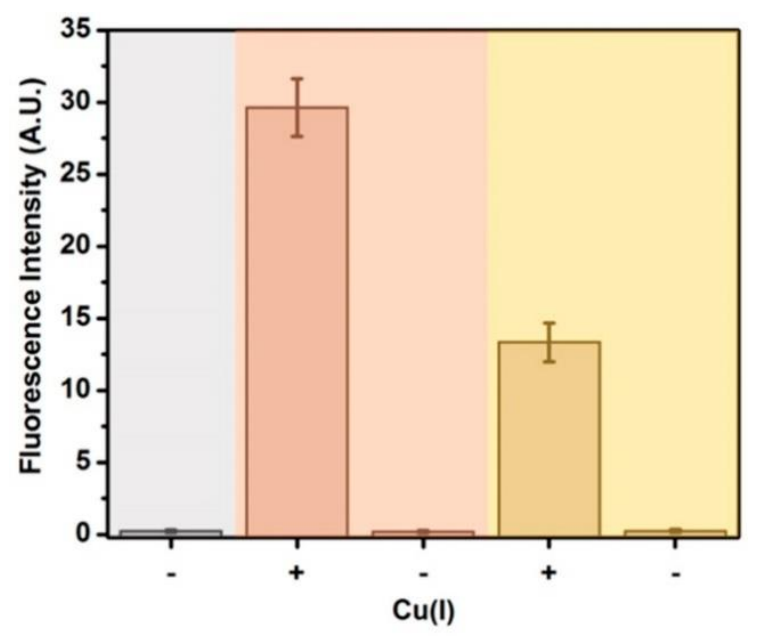

Figure S10. FND fluorescence intensities of unlabelled HFW cells (gray area), azdio-sialoglycoproteinexpressing HFW cells labelled with alkyne-HPGFND (orange area), and HFW cells labelled with antiintegrin $\alpha 5$ antibody and then $100 \mathrm{~nm}$ alkyne-HPGFND (yellow area). The number of cells used in each measurement was 50,000. 
(a)

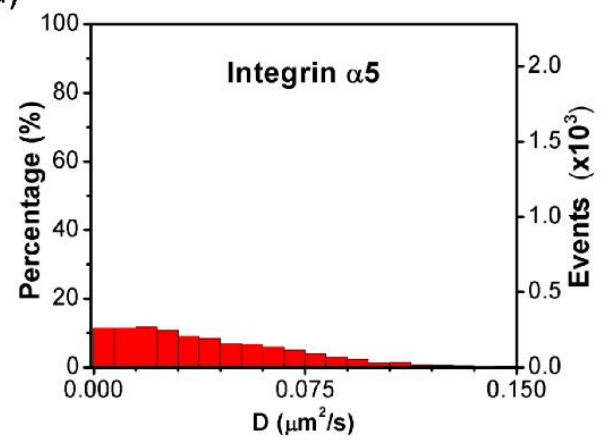

(b)

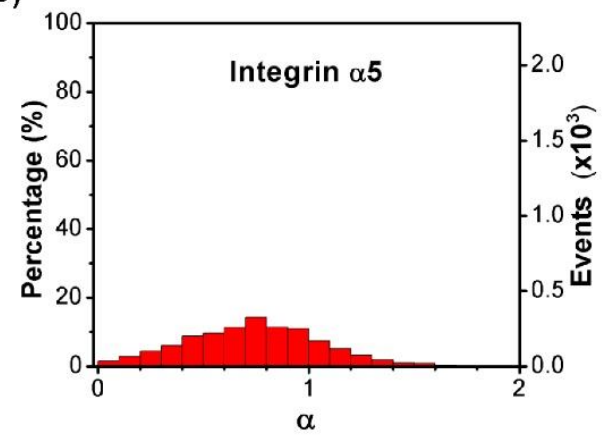

Figure S11. Diffusion coefficients (a) and anomaly parameters (b) of the valid trajectories (longer than 20 continuous frames) recorded in Video S1 for integrin $\alpha 5$. Note that the diffusion coefficients and anomaly parameters were obtained from the MSD data within the time increment of $2 \mathrm{~s}$. 


\section{Video captions}

Video S1. Tracking of alkyne-HPGFND-labelled integrin $\alpha 5$ (red) on living HFW cells by epifluorescence microscopy. The video was produced from a series of images ( $0.104 \mathrm{~s} / \mathrm{image})$ with a rate of $30 \mathrm{frames} / \mathrm{s}$ for $5 \mathrm{~min}$. The alkyne-HPGFNDs used in this work had a nominal diameter of $100 \mathrm{~nm}$.

Video S2. Continuous long-term tracking of alkyne-HPGFND-labelled integrin $\alpha 5$ on/in living HFW cells by confocal fluorescence microscopy. Red and green signals represent alkyne-HPGFND-labelled integrin $\alpha 5$ and Alexa488-WGA-labelled cell membrane, respectively. The video was produced from 52 z-stacked fluorescence/bright-field-merged images, each comprising $\sim 25$ z-sliced images. The total time of the imaging was 3 hours, 58 minutes, and 14 seconds. The alkyne-HPGFNDs used in this work had a nominal diameter of $100 \mathrm{~nm}$.

Video S3. Continuous long-term tracking of alkyne-HPGFND-labelled integrin $\beta 1$ (red) on/in living HFW cells by confocal fluorescence microscopy. The video was produced from $1200 \mathrm{z}$-stacked fluorescence images, each comprising $26 \mathrm{z}$-sliced images. The total time of the imaging was 11 hours, 28 minutes, and 0 seconds. The alkyne-HPGFNDs used in this work had a nominal diameter of $100 \mathrm{~nm}$.

Video S4. Continuous long-term tracking of alkyne-HPGFND-labelled integrin $\beta 1$ (red) on/in living HeLa cells by confocal fluorescence microscopy. The video was produced from $103 \mathrm{z}$-stacked fluorescence images, each comprising $\sim 37 \mathrm{z}$-sliced images. The total time of the imaging was 1 hour, 3 minutes, and 41 seconds. The alkyne-HPGFNDs used in this work had a nominal diameter of $50 \mathrm{~nm}$. 